\title{
The sectional size effect on the deformation behaviour of Inconel 718 at different temperatures
}

\author{
R. Zhao, M. Wan, J.Q. Han ${ }^{\mathrm{a}}$, and B.B. Liu \\ Department of Mechanical Engineering and Science, BeiHang University, Beijing 100083, PR China
}

\begin{abstract}
Inconel 718, as a multiphase super-alloy, is widely used in aeronautics and astronautics industries. In this field, a modified Hall-Petch equation was used to describe the grain size effect on the deformation behaviour of Inconel 718 sheet in uniaxial tension test. There is a piecewise linearity in the $\sigma-\mathrm{d}^{-1}$ curve: With the thickness $\mathrm{t}$ is a constant, the slope changes obviously after a critical $t / d$ ratio, which increases with strain. Moreover, the influence on sectional curve caused by temperature is also an interesting issue. To address that, the sectionalized curve was fitted at different strains and temperatures, and the phenomena of grain size effect in piecewise curve at different temperatures were further explained. A surface model of Inconel 718 was proposed to explain the intrinsic mechanism of different slopes. The research provided an in-depth understanding of the size effect on the deformation behaviour of Inconel 718 at different hot working temperatures.
\end{abstract}

\section{Introduction}

It is well known that the mechanical and physical properties are highly dependent on size effects, and it's of crude importance in thin sheet forming process. There are four main researching aspects of size effect for polycrystalline sheet metals: The thickness of the specimen, the mean diameter of the inner particle, the surface effect and the $\mathrm{t} / \mathrm{d}$ ratio. Beyond that, more and more attentions have been paid to the new orientation of the size effect study. For example, the nanocrystalline metals, the temperature effect and the new size effect testing methods, all of them are based on the fundamental of Hall-Petch relationship, which is used to describe the relationship between the flow stress and its grain size of materials. So it's of critically importance to catch the key point of this classical issue.

Inconel 718 was chosen in this presented article because of its frequently use in aeronautic and astronautic industries, especially in forming process of the turbine blades, micro heating tubes, and thin heat exchange plates. It's usually worked in a hot environment with its excellent high-temperature properties of brazing and forming. However, there's few studies combining the high temperature influence and size effect together with this traditional material. It's necessary to take into account the temperature influence on its grain size effect when we are dealing with the thin sheet forming process of Inconel 718.

Nowadays, many experimental results are available in literatures about the modification of the traditional Hall-Petch dependence of the polycrystalline metallic materials. In particular, it was shown

\footnotetext{
${ }^{\text {a }}$ Corresponding author: hanjinquan_buaa@163.com
}

This is an Open Access article distributed under the terms of the Creative Commons Attribution License 4.0, which permits unrestricted use, distribution, and reproduction in any medium, provided the original work is properly cited. 


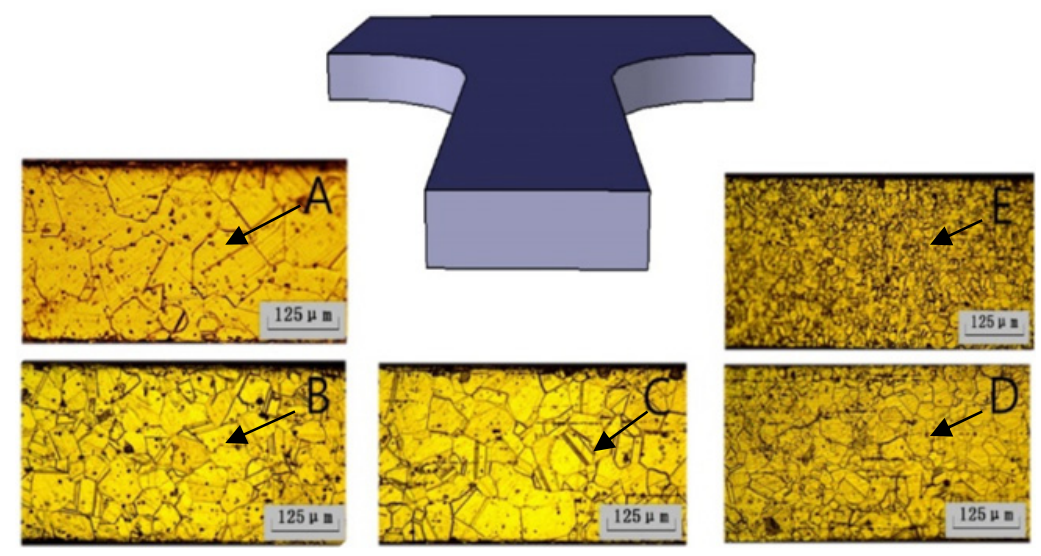

Figure 1. Metallurgical results of different particle size in cross-section.

Table 1. The mean grain size after different heat treatment process.

\begin{tabular}{|c|c|c|}
\hline Specimen ID & Heat treatment temperature $\left({ }^{\circ} \mathbf{C}\right)$ & The mean diameter $(\boldsymbol{\mu}$ m) \\
\hline A & 1100 & 92.371 \\
\hline B & 1050 & 62.849 \\
\hline C & 1000 & 31.663 \\
\hline D & 950 & 19.8 \\
\hline E & - & 13.5 \\
\hline
\end{tabular}

by Keller [1] that a variation of the ratio of thickness (t) to grain size (d) resulted a segment line of stress. It was also reported that this stress reduction appeared when the $\mathrm{t} / \mathrm{d}$ ratio was below a critical value, which depends on stacking fault energy and grain size [2].The purpose of this paper is to describe and discuss the segmental grain size effect dependence of Inconel 718 at different temperatures. First, we use -1 as the proper index number because of its face-centered-cubic structure, of which the fitting results serve as a convincing evidence. Curves of the modified Hall-Petch format at room temperature $25^{\circ} \mathrm{C}, 200^{\circ} \mathrm{C}$ and $400^{\circ} \mathrm{C}$ are presented at different strain levels (ranging from 0.002 to 0.3 ), the trend of the B-spline curves is discussed and compared between different temperatures. To explain the inner mechanism of the variation of the slope, different curves of yield stress vs. the reciprocal of diameter at different temperatures are put together, surface model is applied to elaborate the temperature influence on slope $\mathrm{k}$. The results show a high consistency with the existing research work.

\section{Experiment process}

Inconel 718 sheet with a thickness of $0.3 \mathrm{~mm}$ was used in this presented experiment. The dog-boneshaped samples were handled with a gauge section of $31 \mathrm{~mm}$ in length and $6 \mathrm{~mm}$ in width. In order to reach various inner grain sizes and avoid rolled texture, specimens were under strict heat treatment at temperatures between $950^{\circ} \mathrm{C}$ and $1050^{\circ} \mathrm{C}$ for 20 minutes, then water cooled immediately to remain the high-temperature metallographic structure. After the funance reaches the treatment temperature, there's a heat preservation for 25 minutes, to ensure the particle size grows as we expected. We read the diameters in the cross-section with Heyn's method, choosing five points to get the average result to avoid subjective errors. The samples have a grain size in the range of $13 \mu \mathrm{m}$ and $92 \mu \mathrm{m}$ corresponding to $\mathrm{t} / \mathrm{d}$ ratios between 3.25 and 22.22. The final specifications of prepared specimens are as Fig. 1 and Table 1 shows. 
ICNFT 2015

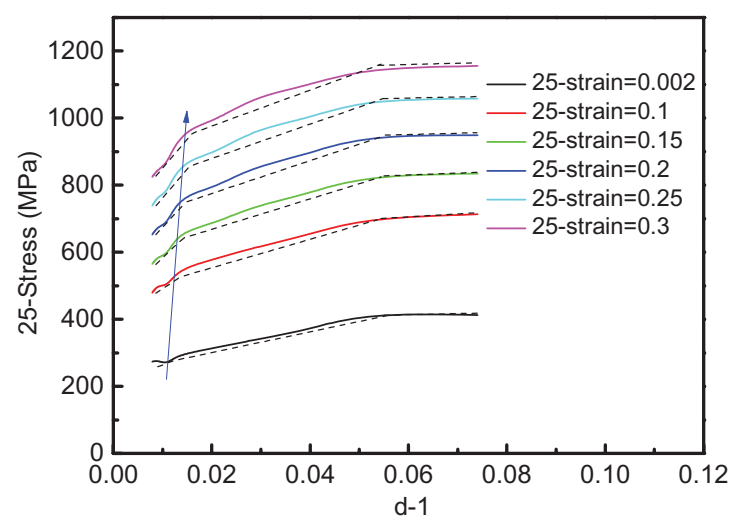

Figure 2. The sectional curves of Inconel 718 at different strain levels at room temperature.

The uniaxial tension test was done on MTS 8800 at temperature between $25^{\circ} \mathrm{C}$ (room temperature) and $400^{\circ} \mathrm{C}$, it was strain rate controlled at a strain rate of $1 \times 10-3$ and started after heat preservation for 10 minutes. Same experiments were done for twice to avoid randomness. Strain measurements were achieved by a high-temperature extensometer.

\section{Results}

Inconel 718 has a strong size effect in deformation process, which could be described by the classical Hall-Petch dependence as Eq. (1) shows:

$$
\sigma=\sigma_{0}+k d^{-1 / 2}
$$

Where $\sigma_{0}$ is the friction stress of mobile dislocations and $\mathrm{k}$ defines the characteristic constant that reflects the impurities and alloying elements effect. The index number [3] which has been discussed as a fit parameter in recent years. Korte [4] suggested that the index number of f.c.c. metals in this formula is near -1 , because the plastic strain $\varepsilon$ corresponding to a single dislocation of effective Burgers vector $\mathrm{b}$ in a finite space of dimension $\mathrm{h}$ is $\mathrm{bh}^{-1}$.

Flow stress curves at room temperature are fitted in Fig. 2, the fitting result confirms Korte's theory [4] at different strain levels from 0.002 to 0.3 . With the thickness $t$ is a constant, the slope of the modified HP curves change after two critical t/d ratios, the smaller $\mathrm{t} / \mathrm{d}$ ratio increases with the true strain, as the blue arrow in Fig. 2 shows. While the larger critical ratio doesn't show an obvious straindependent property. The curves tend to be horizontal at the back part, which means the influence of the particle size on the slope can be ignored when the diameter is small enough compared to the thickness of the sheet.

The sectional curves of Inconel 718 at different strain levels under $200^{\circ} \mathrm{C}$ and $400{ }^{\circ} \mathrm{C}$ are presented in Fig. 3. The linear dependence at $200^{\circ} \mathrm{C}$ is highest, except there's a slight sunken at the middle of the curve, as the black arrow shows. Curves show a contrary tendency to which at room temperature with respect of the slope $\mathrm{k}$ at $400{ }^{\circ} \mathrm{C}$ : The slope expands after the critical $\mathrm{t} / \mathrm{d}$ ratio, and there's only one critical ratio around 10 at $400{ }^{\circ} \mathrm{C}$.

Slopes in the three figures could be described as a strain-independent parameter, while all of the critical number increases with the strain level. The stress is approximately proportional to the reciprocal of the mean diameter, and inversely proportional to the deformation level. 

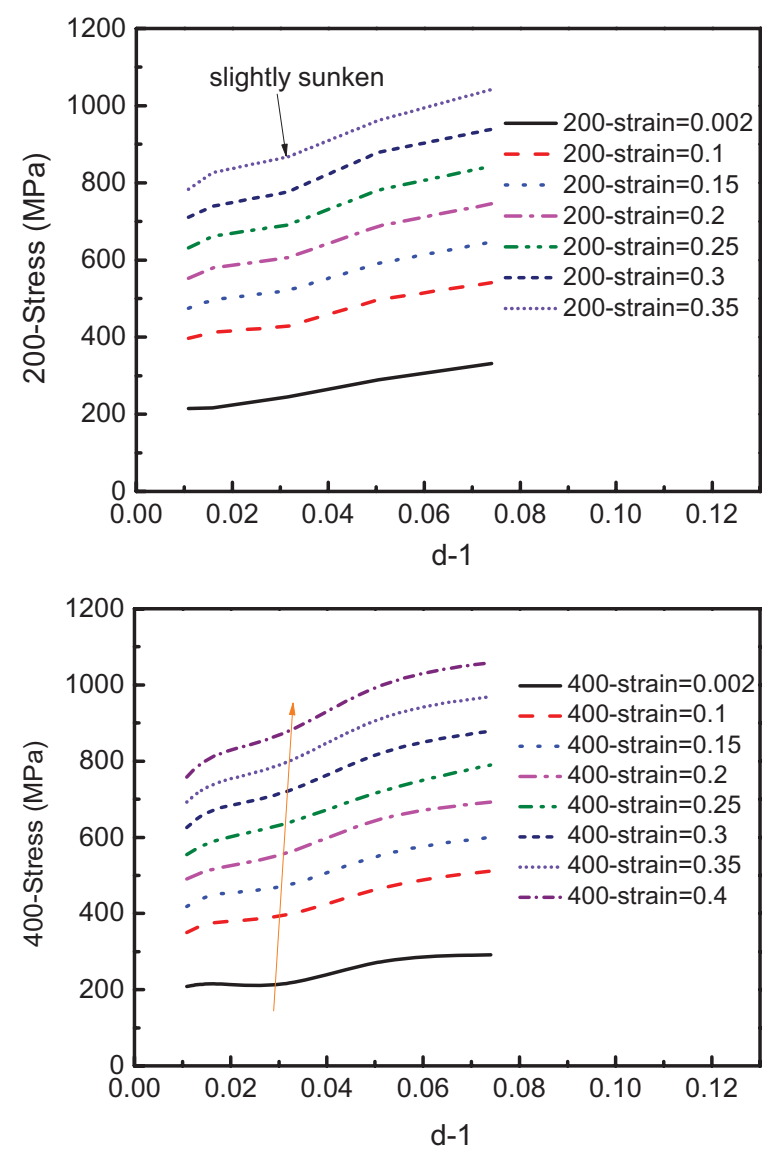

Figure 3. The sectional curves of Inconel 718 at different strain levels under $200^{\circ} \mathrm{C}$ and $400^{\circ} \mathrm{C}$.

\section{Discussion}

\subsection{Sectional phenomenon from multi-crystals to poly-crystals at room temperature}

The thickness of the specimen sheets is a constant value of $0.3 \mathrm{~mm}$ in this presented article, so it's equivalent for us to decide the $\mathrm{t} / \mathrm{d}$ ratio by determining the mean diameter of the inner particle size.

As mentioned in Fig. 2, two critical measurements are found at the room temperature deformation process. A similar sectional phenomenon is discovered in polycrystalline nickel [1]. The cause of critical number and sectional phenomenon lies in that the stress decreases when the particle size becomes larger, which could be understood by the surface effect of poly-crystals: the dislocation nearby the free surface can emerge easily through the latter. While the grain boundaries act as obstacles, reducing the mean free path of the dislocations, it also has a relatively low dislocation density near the free surfaces. As a result, the gradient distance of poly-crystals, set as L here representing the stress or dislocation gradient distance, becomes smaller than in single-crystal due to the grain boundary obstacle effects.

\subsection{Influence of temperature on sectional slopes}

There's different sectional appearance from room temperature to $400{ }^{\circ} \mathrm{C}$ at different strain levels in Fig. 2 and Fig. 3. The slopes of the curves are obviously larger at room temperature with the increase of 


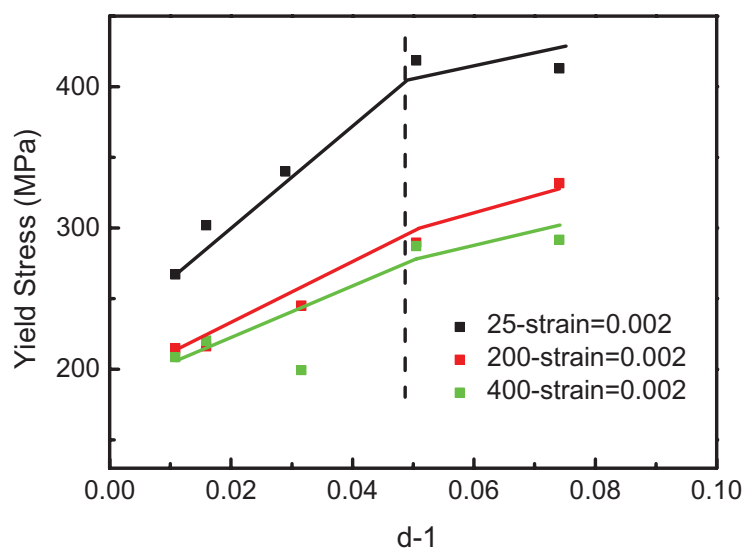

Figure 4. The $\sigma_{0.2}-\mathrm{d}^{-1}$ curves at different temperatures, showing an obvious sectional phenomenon of the slope $\mathrm{k}$.

the particle size at room temperature, whatever the strain level is. Furthermore, the slope k remains the same in the deformation process, which means the slope at a high strain level is similar with what in a low strain level at a specific temperature. Besides that, the critical ratio is about 30 at room temperature, which becomes 12 at $200{ }^{\circ} \mathrm{C}$ and 10 at $400^{\circ} \mathrm{C}$, and the critical ratios increase slightly with the increasing strain level. All of the above-mentioned phenomenon is related to the variation of slope $\mathrm{k}$. To study the influence factor of the slope k, a classical theory is proposed by Armstrong [5] reads:

$$
k=m^{2} \tau_{c} r^{1 / 2} .
$$

Where $\mathrm{m}$ is the orientation factor related to the number of activated slip systems, $\tau_{c}$ stands for the critical resolved shear stress (CRSS) required for operation of a dislocation source and $r$ denotes the distance from the nearest dislocation piled-up to the dislocation source in the adjacent grain. With the increasing temperature, both the critical resolved shear stress $\tau_{c}$ and the number of the active slip systems reduces, which in turn, decreases the $\mathrm{k}$.

To show the variation tendency of slope influenced by temperature, we put $\sigma_{s}-\mathrm{d}^{-1}$ curves together in Fig. 4. There's a critical line (indicated as a black dotted-line in Fig. 4) located at the middle of the curves, before which the slope $\mathrm{k}$ decreases obviously with increasing temperature, and the slope keeps a constant after the critical line. Similar result has been found by Hauser [6] and Ono [7]. From which could we contend the slope decreases slower with the raising temperature.

The slope decreases much more obviously with larger particle size at a high temperature, which could be also explained by the surface effect. It's interesting to study the high-temperature sectional curves first discovered in Inconel 718 continuely. In order to identify the critical number, mechanical behaviors of grain boundary and grain interior, property of surface layer and volume fraction of surface layer should be determined [8], which is very complicated and costly, and of crouse, a worthy work.

\section{Conclusion}

This presented article mainly focuses on the influence of temperature on sectional grain size effect of Inconel 718. Our work can be summarized by the three main following points:

- The stress decreases with the increasing particle size, which has been explained by the surface model.

- There's a segment phenomenon in the deformation process of Inconel 718 at room temperature. With the increase of the temperature, the segment still exists, but the variation tendency of the 


\section{MATEC Web of Conferences}

slope $\mathrm{k}$ changes: The slope increases after the critical $\mathrm{t} / \mathrm{d}$ ratio, instead of decreasing clearly at room temperature, and the linearity is the most obvious at $200^{\circ} \mathrm{C}$. All of the critical $\mathrm{t} / \mathrm{d}$ ratios increase with the true strain.

- There's also a critical measurement of $20 \mu \mathrm{m}$ in particle size comparing with yielding stress at different temperatures, after which the slope seems temperature-independent. The slope decreases with the temperature before this critical number, which could be also explained by the influence of surface model.

The author would like to thank the Research Project (No. 11600002014104001) and The Fundamental Research Funds for the Project in National Laboratory for Aeronautics and Astronautics in Beihang for the support given to this research, and this work is also supported by Graduate Innovation Fund of Beihang University in 2014.

\section{References}

[1] C. Keller, E. Hug, Mater Lett, 62, 1718-1720 (2008)

[2] P.J.M. Janssen, Th.H. de Keijser, M.G.D. Geers, Mater. Sci. Eng. A, 419, 238-248 (2006)

[3] D.J. Dunstan, A.J. Bushby, Int. J. Plasticity, 53, 56-65 (2014)

[4] S. Korte, W.J. Clegg, Philos. Mag., 91, 1150-1162 (2011)

[5] R. Armstrong, I. Codd, R.M. Douthwaite, N.J. Petch, Philos. Mag., 7, 45-58 (1962)

[6] F.E. Hauser, P.R. Landon, J.E. Dorn, Trans. TMS-AIME, 206, 589-593 (1956)

[7] N. Ono, R. Nowak, S. Miura, Mater Lett, 58, 39-43 (2004)

[8] Q. Wang, X.H. Dong, H.M. Zhang, H.Z. Li, Y. Shen, T. Nonferr. Metal. Soc., 23, 1428-1434 (2013) 